\title{
The development of generalized synchronization on complex networks
}

\author{
Shuguang Guan, ${ }^{1,2}$ Xingang Wang, ${ }^{1,2}$ Xiaofeng Gong, ${ }^{1,2}$ Kun Li, ${ }^{1,2}$ and C.-H. Lai ${ }^{2,3}$ \\ ${ }^{I}$ Temasek Laboratories, National University of Singapore, Singapore 117508 \\ ${ }^{2}$ Beijing-Hong Kong-Singapore Joint Center of Nonlinear and Complex systems (Singapore), Singapore 117508 \\ ${ }^{3}$ Department of Physics, National University of Singapore, Singapore 117543
}

(Dated: October 28, 2018)

\begin{abstract}
In this paper, we investigate the development of generalized synchronization (GS) on typical complex networks, such as scale-free networks, small-world networks, random networks and modular networks. By adopting the auxiliary-system approach to networks, we show that GS can take place in oscillator networks with both heterogeneous and homogeneous degree distribution, regardless of whether the coupled chaotic oscillators are identical or nonidentical. For coupled identical oscillators on networks, we find that there exists a general bifurcation path from initial non-synchronization to final global complete synchronization (CS) via GS as the coupling strength is increased. For coupled nonidentical oscillators on networks, we further reveal how network topology competes with the local dynamics to dominate the development of GS on networks. Especially, we analyze how different coupling strategies affect the development of GS on complex networks. Our findings provide a further understanding for the occurrence and development of collective behavior in complex networks.
\end{abstract}

PACS numbers: 05.45.Xt,89.75.Hc

\section{INTRODUCTION}

Synchronization in coupled chaotic oscillators has been extensively studied in the past twenty years [1], including complete synchronization [2], generalized synchronization [3, 4, 5, 6, 7], and phase synchronization (PS) [8], etc. In CS, the dynamics of two coupled systems totally coincide with each other; in GS, certain functional relation exists between the dynamics of two coupled systems which are usually nonidentical. Moreover, PS is a weaker synchronization form in which the phases of two oscillators can be locked while their amplitudes remain uncorrelated and chaotic. Recently, the study of synchronization has been extended to the area of complex networks $[9,10,11,12,13,14,15,16,17,18,19$, $20,21,22,23$. For example, synchronization on small-world networks [9, 10, 11, 12], scale-free networks [13, 14, 15], modular networks [16, 17, 18, 19], weighted networks [20], and gradient networks [21], have been investigated. These studies aim to explore the interplay between network topology and dynamics on network. They are important for us to understand the real situations in complex systems comprising interacting elements in nature and human society.

In literatures, there are two main approaches studying synchronization in complex networks. One approach is to study synchronization of phase oscillators on complex networks, i.e., the generalized Kuramoto models [12, 19, 22, 23, 24]. In this model, the node dynamics is very simple which is governed by an ideal phase oscillators, $\dot{\phi}=\omega$, where $\omega$ is the frequency. The heterogeneity in the node dynamics can be modeled by assigning different, usually random, frequencies to different phase oscillators. The generalized Kuramoto models in complex networks have the advantage that they can still be treated analytically in many aspects [19, 22]. The second approach is to study the synchronizability of complex networks through the master stability function [11, 25]. To apply this approach, the node dynamics in complex networks are assumed to be identical, and then the theory of master stability function provides a general mathematical framework to relate the synchronizability of a network to the spectral properties of the corresponding coupling matrix [11, 14, 17, 20, 26].

The above two approaches allows an analytical and therefore relatively more complete understanding of synchronization in complex networks. Nevertheless, in realistically physical or biological situations the node dynamics can conceivably be much more complicated, and in general be different from each other. The key point is that the possible collective behaviors for coupled nonidentical oscillators should be of the type of GS rather than CS and PS. It is thus of great importance to investigate the collective behaviors on complex networks where the local node dynamics might be different from each other. In this paper, we present a systematical study on GS phenomenon in various complex networks. Our particular attention is paid to the development of GS in networks. For typical complex networks, such as scale-free networks, smallworld networks, random networks and modular networks, interestingly, we find that GS generally occurs in these networks, regardless of whether the node dynamics are identical or nonidentical. For coupled identical oscillators on complex networks, a general bifurcation path toward the final global CS is shown as: non-synchronization $\rightarrow$ PGS $\rightarrow$ GS $\rightarrow$ PCS $\rightarrow$ CS. Here, PGS and PCS stand for partial GS and partial CS, respectively. We further study how the network topology competes with the local dynamics to affect the development of GS on networks. It is shown that for linearly coupled oscillators on heterogeneous networks, GS usually starts from the hub nodes and then spreads to other nodes; while on homogeneous networks, GS generally starts from the nodes whose local dynamics are less chaotic in the sense that the largest Lyapunov exponents have relatively smaller values. Moreover, we analyze how different coupling strategies can affect the development of GS on complex networks, a point which has not been addressed before.

In processing this paper, we noticed a recent work which has reported GS phenomenon in scale-free networks [27]. Compared with the results in Ref. [27], the present work is different in the following aspects. Firstly, in our work GS is 
systematically investigated on various complex networks, including scale-free networks, random networks, small-world networks and modular-networks; while in Ref. [27], GS is mainly studied on a very special network, i.e., the scalefree networks with tree-like structure. Secondly, the present work characterizes the complete bifurcation path for coupled identical oscillators on complex networks, i.e., from non-synchronization to global CS, including the transition regimes; while in Ref. [27], global CS has not be achieved. Thirdly, the present work investigates the development of GS for coupled nonidentical oscillators, either parametrically different or physically different; while Ref. [27] does not consider this general setting where the occurrence of GS is naturally justified. Finally, in the present work, we discuss the effect induced by different coupling strategies on the development of GS on network. Especially, we analyze and explain the different observations regarding the GS evolution on networks in this work and in Ref. [27]. In fact, in many aspects the current study deepens and widens the work in Ref. [27], and can offer more thorough and comprehensive insight in understanding GS phenomenon on complex networks.

This paper is organized as follows. In Sec. II, the methods to characterize GS and CS on complex networks are described. In Sec. III, GS of coupled identical oscillators is studied on complex networks. The complete bifurcation path from non-synchronization to global CS is studied and the dynamical features in the transition regimes are characterized. In Sec. IV, GS of coupled nonidentical oscillators is further studied on complex networks. Our interest is focused on how the development of GS on networks can be affected by network topology and the local dynamics. In Sec. V, the effect of different coupling strategies on the development of GS on complex networks is analyzed. Especially, GS is observed in coupled system with hybrid oscillators even when their local dynamics are physically different. Finally, a summary ends this paper in Sec. VI.

\section{APPROACHES CHARACTERIZING GS AND CS ON NETWORKS}

The auxiliary-system approach has been extensively used to detect GS in two coupled chaotic systems [5]. Here, we can extend it to detect GS on complex networks. The key observation is that, for any given node in a network, the coupling from other nodes can be regarded as a kind of "driving." In particular, we consider the following linearly coupled identical oscillators on a network:

$$
\dot{\mathbf{x}}_{i}=\mathbf{F}_{i}\left(\mathbf{x}_{i}\right)-\varepsilon \sum_{j} a_{i j}\left(\mathbf{x}_{i}-\mathbf{x}_{j}\right),
$$

for $i=1, \ldots, N$, where $\mathbf{x}_{i}$ denotes the dynamical variables of node $i, \mathbf{F}_{i}\left(\mathbf{x}_{i}\right)$ is the local vector field governing the evolution of $\mathbf{x}_{i}$ in the absence of interactions with other nodes, $a_{i j}$ is the element of the network adjacency matrix $\mathbf{A}\left(a_{i j}=1\right.$ if there is a link between node $i$ and node $j, a_{i j}=0$ otherwise, and $a_{i i}=0$ ), and $\varepsilon$ is the coupling strength. To apply the auxiliary-system approach, we consider a replica for each oscillator in the original network:

$$
\dot{\mathbf{x}}_{i}^{\prime}=\mathbf{F}_{i}\left(\mathbf{x}_{i}^{\prime}\right)-\varepsilon \sum_{j} a_{i j}\left(\mathbf{x}_{i}^{\prime}-\mathbf{x}_{j}\right),
$$

for $i=1, \ldots, N$. Note that the driving variable $\mathbf{x}_{j}$ is identical for both Eqs. (1) and (2). If, for initial conditions $\mathbf{x}_{i}(0) \neq \mathbf{x}_{i}^{\prime}(0)$, we have $\left|\mathbf{x}_{i}(t)-\mathbf{x}_{i}^{\prime}(t)\right| \rightarrow 0$ as $t \rightarrow \infty$, node $i$ then is entrained in the sense that its dynamics is no longer sensitive to initial conditions. In other words, there is GS relation between $\mathbf{x}_{i}$ and $\mathbf{x}_{j}$ for $j=1, \ldots, N$. Numerically, we can examine the following local distance of GS between a node and its auxiliary counterpart:

$$
d(\varepsilon, i)=\frac{1}{t_{2}-t_{1}} \sum_{t_{1}}^{t_{2}}\left|\mathbf{x}_{i}(t)-\mathbf{x}_{i}^{\prime}(t)\right|
$$

where $t_{1}$ is chosen to be larger than the typical transient time of the local dynamics $\mathbf{F}_{i}\left(\mathbf{x}_{i}\right)$. For oscillators on complex networks, GS may be gradually developed with the increase of the coupling strength. To characterize the development of GS on networks, we can define the distance of global GS as $l_{g}(\varepsilon)=\langle d(\varepsilon, i)\rangle$. Here, $\langle\cdot\rangle$ denotes the spatial average over all nodes. If $l_{g}=0$, global GS has been achieved between any two pair of oscillators on the whole network.

For coupled identical oscillators on complex networks, CS is generally expected to take place as long as the network connectivity is not too sparse. To characterize CS, we can define $l_{c}(\varepsilon)$ as the distance of global CS, which measures the distance between the dynamics of all oscillators and their average, i.e.,

$$
l_{c}(\varepsilon)=\frac{1}{t_{2}-t_{1}} \sum_{t_{1}}^{t_{2}}\langle|\mathbf{x}-\langle\mathbf{x}\rangle|\rangle,
$$

where the meaning of $t_{1}$ and $t_{2}$ is the same as that in Eq. (3). If $l_{c}=0$, global CS has been achieved on the whole network.

For individual node dynamics, we choose the chaotic Lorenz oscillator:

$$
\mathbf{F}_{i}\left(\mathbf{x}_{i}\right)=\left[10\left(x_{i}-y_{i}\right), r_{i} x_{i}-y_{i}-x_{i} z_{i}, x_{i} y_{i}-(8 / 3) z_{i}\right]^{T},
$$

where $\mathbf{x}_{i} \equiv\left(x_{i}, y_{i}, z_{i}\right)$ are the state variables of the Lorenz oscillator. Note that in studying GS, the local dynamics could be different from each other. This can be modelled by setting different $r_{i}$ values for Lorenz oscillators in the network. Without losing generality, the coupling between two nodes in Eq. (1) is through the $x$ variable. For convenience, in the present work, we use two kinds of node indices: $i_{d}$ and $i_{r}$. In the first index, we order the degrees of the network so that $i_{d}=1 \mathrm{de}$ notes the node with the largest degree, $i_{d}=2$ is for the node with the second largest degree, and so on. In the second index, we rank the Lorenz oscillators in the network according to their $r_{i}$ values, i.e., $i_{r}=1$ denotes the node with the largest parameter $r, i_{r}=2$ is for the node with the second largest parameter $r$, and so on. These two indices of nodes are used throughout the paper. 


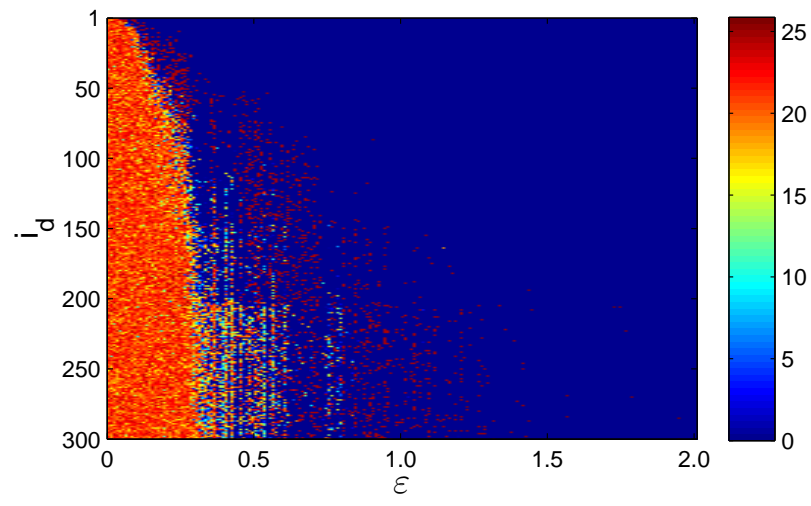

FIG. 1: (Color online) Color-map of $d(\varepsilon, i)$ in the two-dimensional parameter space $(\varepsilon, i)$, characterizing the development of GS for 300 identical chaotic Lorenz oscillators on a scale-free network. The network is the BA model with $m_{0}=m=4$ [13]. We see that with the increase of coupling strength, GS can be achieved gradually from the hubs and then spread to the rest of the network.

\section{COUPLED IDENTICAL OSCILLATORS: A GENERAL PATH TOWARD GLOBAL CS}

A. Path towards CS on scale-free networks
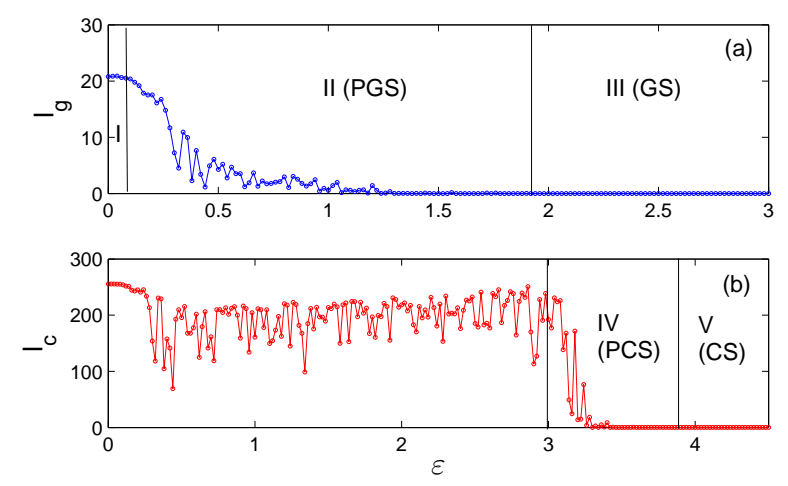

FIG. 2: (Color online) Characterizing the path towards global CS on scale-free network. (a) The distance of global GS vs the coupling strength. (b) The distance of global CS vs the coupling strength. Numerically, if $l_{g}\left(l_{c}\right)<0.001$, global GS (CS) is regarded to have been achieved. The notations of the regimes in this figure are used throughout this paper.

Previously, in the study of synchronization of two coupled chaotic oscillators, it is found that CS generally takes place when the coupled oscillators are identical, while GS usually occurs in systems of coupled nonidentical oscillators. This observation is accurate for most cases except for some specially designed coupling strategies [7]. However, this generally accepted view turns out to be not true when synchronization is studied on complex networks. In this section, we investigate the occurrence of GS for coupled identical chaotic oscillators on various complex networks. Our particular interest is focused on the complete bifurcations from initial nonsynchronization towards the final global CS with the increase of the coupling strength. By applying auxiliary-system approach, interestingly, we find that GS generally exists in coupled identical oscillators on various complex networks, including scale-free networks, random networks, small-world networks and modular networks.

As an example, we first study the bifurcation route towards global CS for 300 coupled chaotic Lorenz oscillators on a scale-free network. In this case, $r_{i}=28$ in Eq. (5) for all nodes, i.e., all oscillators are identical. Intuitively, with the increase of coupling, we expect that the coupled chaotic oscillators will finally achieve global CS. The surprising finding here is that before the system achieves the final global CS, there exists another synchronization regime, i.e., the GS regime, which usually occurs with much smaller coupling strength compared with CS. To illustrate the GS phenomenon on scale-free network, we plot the color-map of the distance matrix $d\left(\varepsilon, i_{d}\right)$ in Fig. 1. From Fig. 1, three distinct regimes of coupling can be identified from the viewpoint of GS. When the coupling strength is small, $d\left(\varepsilon, i_{d}\right)$ are greater than 0 for all nodes, showing that the system is in the non-synchronous state. On the other hand, when the coupling strength is large enough, $d\left(\varepsilon, i_{d}\right)$ are 0 for all nodes, showing that all oscillators have been entrained and the coupled system is in the global GS state according to the auxiliary system approach criterion. Between these two regimes, it is the regime of partial GS, where part of the oscillators have been entrained but the others have not. Obviously, this regime is a transition stage between nonsynchronous state and the global GS state.

To quantitatively characterize the GS regime for coupled chaotic oscillators on scale-free network, in Fig. 2(a) we plot the distance of global GS $l_{g}$ vs the coupling strength. From this figure, three regimes of coupling can numerically be identified. On the other hand, for coupled identical oscillators, CS is generally expected to occur as long as the connections of the network are dense enough. It is found that usually CS requires larger coupling strength than GS. This implies that GS is a weaker synchronization form compared with CS on complex networks, as in the situation of coupled two-oscillator systems. To characterize CS in the network, we plot the distance of global CS $l_{c}$ vs the coupling strength in Fig. 2(b). We find that before CS is achieved, there is also a transition stage, i.e., the partial CS regime. Combining all the results in Fig. 2, we can identify five dynamical regimes on the path towards global CS on the specific scale-free network as follows. I: $\varepsilon \leq 0.1$, the non-synchronization regime; II: $0.1<\varepsilon \leq 1.8$, the PGS regime; III: $1.8<\varepsilon<3.0$, the global GS regime; IV: $3.0<\varepsilon<3.8$, the PCS regime; V: $\varepsilon>3.8$, the global CS regime. These regimes thus characterize the complete bifurcations from non-synchronization state to global CS state for coupled identical oscillators on scale-free networks. 

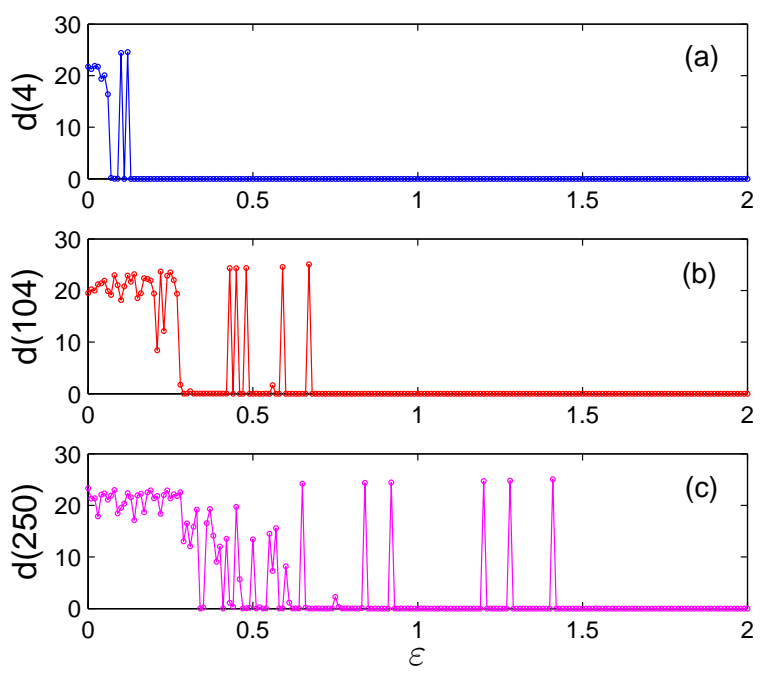

FIG. 3: (Color online) Typical routes toward final GS for Lorenz oscillators in scale-free network. From (a) to (c), $d\left(\varepsilon, i_{d}\right)$ versus $\varepsilon$ for $i_{d}=4,104$, and 250, respectively. We observe that nodes usually oscillate between the entrained state (GS) and the unentrained state on the way toward final global GS state as the coupling strength is increased.
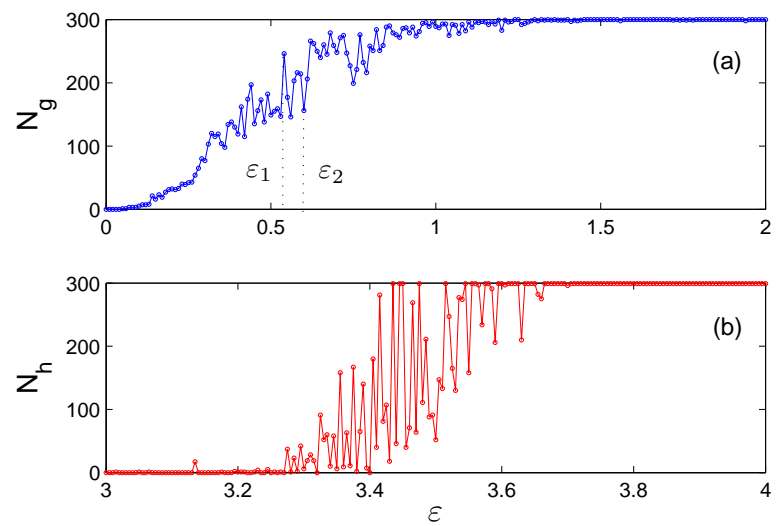

FIG. 4: (Color online) Characterizing the dynamic and oscillatory nature in the PGS and PCS regimes on a scale-free network. (a) The number of nodes which have achieved GS vs the coupling strength. (b) The number of nodes which have achieved CS with the hub (the node with the largest degree) vs the coupling strength.

\section{B. Dynamic and oscillatory transition to global synchronization}

In the above we have shown that there are two transition regimes, i.e., the PGS regime and PCS regime, before the coupled system achieves global GS and CS, respectively. In fact, these two regimes are important to obtain information about how synchronization is generated and spread on a network. As shown in Fig. 1, with the increase of coupling strength, (a)
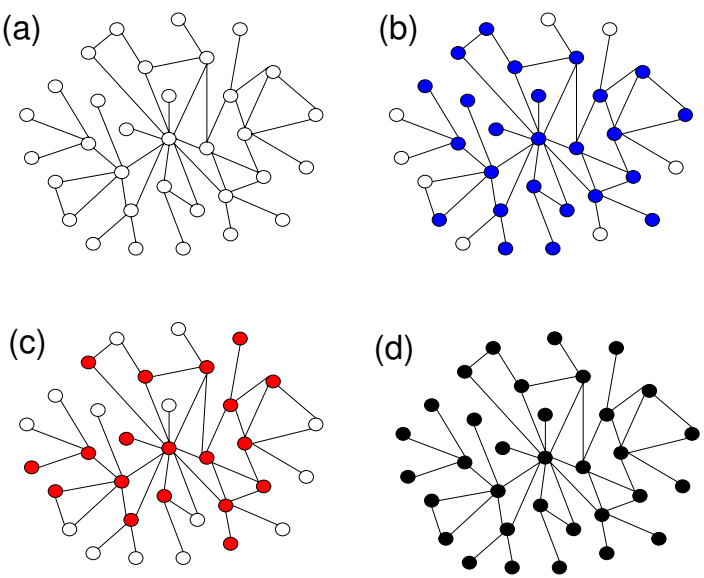

FIG. 5: (Color online) A schematic visualization of the oscillatory path toward global GS shown in Fig. 4(a). (a) Non-synchronous state at $\varepsilon=0$. (b) PGS state at $\varepsilon_{1}=0.55$. About 80 percent nodes in the network (nodes with blue color) are entrained. (b) PGS state at $\varepsilon_{2}=0.61$. About 50 percent nodes in the network (nodes with red color) are entrained. (d) Global GS state at $\varepsilon=3$.

usually the hubs of scale-free network become entrained first. Then more and more nodes are entrained with further increase of the coupling strength. At $\varepsilon \approx 0.3$, a giant cluster of synchronous nodes in the sense of GS has been formed. The most important dynamic feature in the PGS regime is that nodes may oscillate between the entrained state and the unentrained state with the increase of the coupling strength. As shown in Fig. 3, if we focus on a specific node, we find that its path toward global GS is generally oscillatory. Especially, for those nodes with small degrees, the path could be extremely oscillatory as shown in Fig. 3(c). As a consequence, in the PGS regime, the size of the synchronous giant cluster does not increase monotonically, instead it increases in an oscillatory manner with the increase of the coupling strength, as shown in Fig. 4(a). Similar phenomenon has been observed in the PCS regime. For example, in Fig. 4(b), we plot the number of nodes which have achieved CS with the hub (with the largest degree). This figure actually characterizes the dynamical features for regime IV shown in Fig. 2(b). It is found that the size of the synchronous cluster oscillates drastically on the way toward global CS with the increase of the coupling strength.

Recently, the path towards global PS in generalized $\mathrm{Ku}$ ramoto model has been studied in Ref. [23]. It is found that for phase oscillators with the simplest local dynamics, once a phase oscillator becomes frequency locked, it will never escape from the synchronous cluster. Thus in the PS situation the size of the synchronous cluster increases monotonically with the increase of the coupling strength. Such a path toward global synchronization can be called as static and monotonic. In the present work, we demonstrate a fundamentally different scenario towards global synchronization for coupled chaotic oscillators on complex networks. The most important feature 
of the path is that the development and spreading of synchronization on networks is a dynamic process during which oscillators may frequently oscillate between the non-synchronous state and the synchronous state. This implies that the size of the synchronous cluster might be sensitive to the perturbation of the coupling strength in the transition regimes. To visualize the dynamic feature of the development of synchronization on networks, a schematic figure has been plotted in Fig. 5 for a scale-free network. Our results suggest that in more realistic situations, the path towards global synchronization on complex networks could be dynamic and oscillatory rather than static and monotonic.

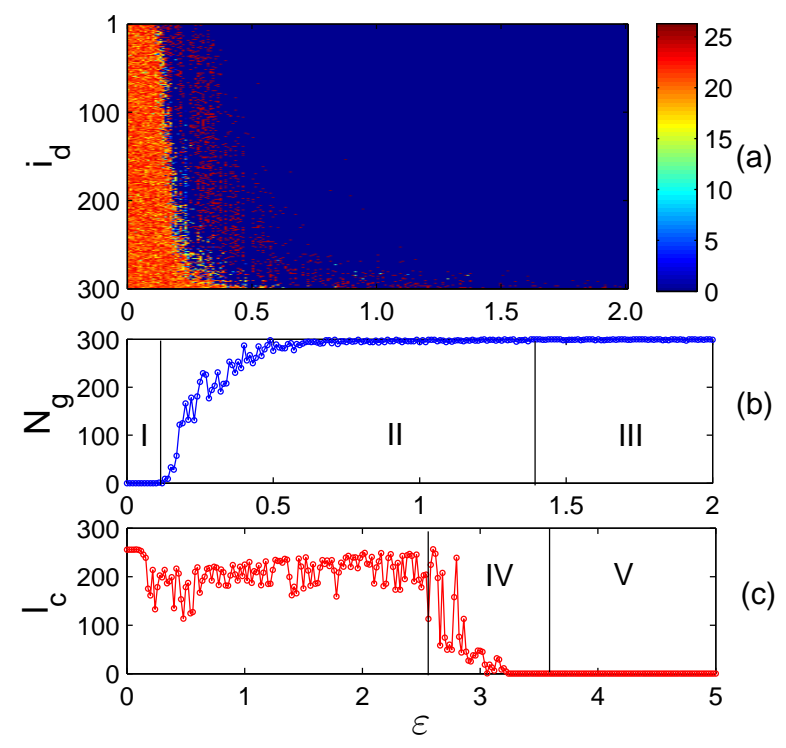

FIG. 6: (Color online) Characterizing the development of GS and the path toward global CS of 300 coupled identical Lorenz oscillators on a random network with average degree 10. (a) The color-map of $d(\varepsilon, i)$ in the two-dimensional parameter space $(\varepsilon, i)$. (b) The number of nodes which have achieved GS vs the coupling strength. (c) The distance of global CS vs the coupling strength.

\section{Path toward global CS on other types of complex networks}

In the above we have characterized the path towards global CS for coupled identical chaotic oscillators on scale-free network, which is characterized by a heterogeneous degree distribution. We have also investigated synchronization path for coupled identical chaotic oscillators on other typical complex networks. Specifically, the networks studied include the following: (1) A random network consisting of 300 nodes. The average degree is 10. (2) A small-world network, which is obtained by rewinding 20 links in a regular network consisting of 100 nodes and each node has 10 nearest neighbor connections [9]. (3) A modular network consisting of 100 nodes which is evenly divided into 5 modules. Inside each module nodes are fully connected. Any two nodes in different modules have probability $p=0.01$ to connect each other. (4) As a special case of complex networks, a regular network consisting of 100 nodes and each node has 10 nearest neighbor connections.

The pathes towards global CS on these typical complex networks have been characterized as for scale-free networks. Here, we only show one example in the case of random network. In Fig. 6, the bifurcation route to global CS for 300 identical Lorenz oscillators on a random network is characterized. Comparing Fig. 6(a) with Fig. 1, we can see that the development of GS on random network is different from the situation on scale-free network. Mainly, most nodes are entrained at approximately the same coupling strength. This is consistent with the fact that random network has approximately homogeneous degrees and thus no extraordinary hubs exist as in scale-free networks. Although the development of GS on different networks depends on the network topologies, qualitatively, the path toward global GS is the same as shown in Fig. 1 and Fig. 6. In fact, for other types of complex networks, we have similar observations, as in the scale-free network and random network. Based on these studies, we can draw the following conclusions. (1) For coupled identical chaotic oscillators, whether the network is homogeneous or heterogeneous, a general bifurcation path towards global CS exists with the increase of coupling strength, i.e, non-synchronization $\rightarrow$ PGS $\rightarrow$ GS $\rightarrow$ PCS $\rightarrow$ CS. (2) Usually, global GS needs relatively smaller coupling strength to achieve compared with global CS. Sometimes, the latter requires ten times larger coupling strength to achieve than the former. This shows that GS is a weaker synchronization form on complex networks compared with CS, and is consistent with the observation in coupled two-oscillator systems. (3) Typically, the coupled system undergoes a dynamic and oscillatory transition to achieve global synchronization, such as global GS or global CS. During the PGS or PCS regime, oscillators may frequently join in and escape from the giant synchronous cluster. This leads to that the size of synchronous giant cluster increases in an oscillatory manner with the increase of the coupling strength. In some circumstances, the transition regimes cover quite wide range of coupling strength, reflecting that there exists intense interaction among nodes in achieving global synchronization on complex networks.

\section{COUPLED PARAMETRICALLY DIFFERENT OSCILLATORS: TOPOLOGY VS LOCAL DYNAMICS}

So far, most of the works on synchronization in complex networks study the coupled identical oscillators, or coupled simple phase oscillators of Kuramoto type. However, in realworld networks, the interacting components are in principle different from each other. For example, in neuron networks any two pair of neurons cannot be exactly the same. This raises an important question: is it possible for complex networks with different local dynamics achieve GS, or certain coherence? If so, how network topologies affect the GS? In this section, we will investigate synchronization of coupled nonidentical oscillators on complex networks. Our particular interest is to reveal how the development of GS on networks is dominated by the interplay between network topology and 


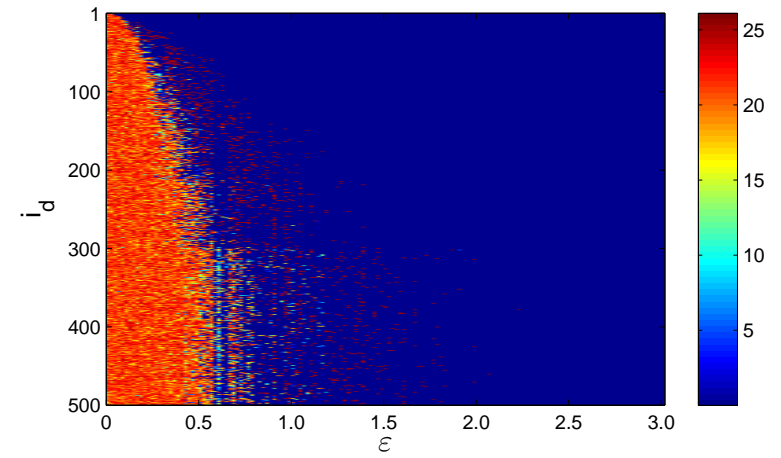

FIG. 7: (Color online) Color-map of $d\left(\varepsilon, i_{d}\right)$ in the two-dimensional parameter space $(\varepsilon, i)$, characterizing the development of GS for 500 non-identical Lorenz chaotic oscillators on a scale-free network. The network is the BA model with $m_{0}=m=3$ [13]. The development of GS in the network is similar to the situation shown in Fig. 1.

the local dynamics.

As in the case of coupled system with two different oscillators, we find that CS is generally forbidden for coupled nonidentical oscillators on complex networks. Instead, we observe that GS generally occurs in such coupled systems on networks. In our study, the Lorenz oscillators, i.e., Eq. (5), are made different on network by randomly setting parameter $r_{i}$ in the interval $[28.0,30.0]$. By applying auxiliary system approach, we can plot the color-map of the local synchronization distance $d\left(\varepsilon, i_{d}\right)$ in the two-dimensional parameter plane $\left(\varepsilon, i_{d}\right)$. As an example, Fig. 7 illustrates the development of GS for Lorenz oscillators with parameter mismatches on a scale-free network. It is shown that with the increase of coupling strength, nodes in the network are entrained first from the hubs and gradually spread to other nodes. When the coupling strength is large enough, all nodes in the network are entrained indicating global GS has been achieved. This situation is similar to the case of coupled identical oscillators due to the fact that the parameter mismatches here are small. Moreover, for coupled nonidentical oscillators, the dynamical feature of the route toward global GS is essentially the same as in the case of identical oscillators.

The interplay between network dynamics and network topology is the key to understand collective behaviors on complex networks. Previously, many works have revealed how network topology affects the network synchronizability under the setting of coupled identical oscillators. For nonidentical oscillators, there are two factors affecting the development of GS on complex networks, i.e., network topology and heterogeneity in the local dynamics. The effect of heterogeneity in the local dynamics, if they are chaotic, on the development of GS remains to be a challenging problem. In the two preceding sections, we have shown that for scale-free networks, GS typically starts from the hubs, and then spreads to others nodes with relatively smaller degrees. In this case, heterogeneity in the degree distribution appears to be the main factor governing the development of GS. An interesting issue is then that, for networks with homogeneous degree distributions, such as

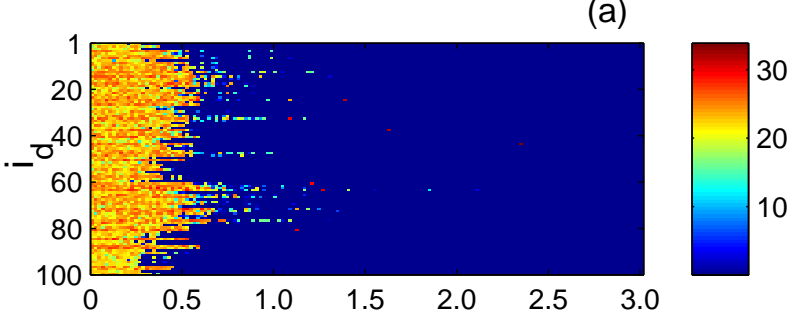

(b)

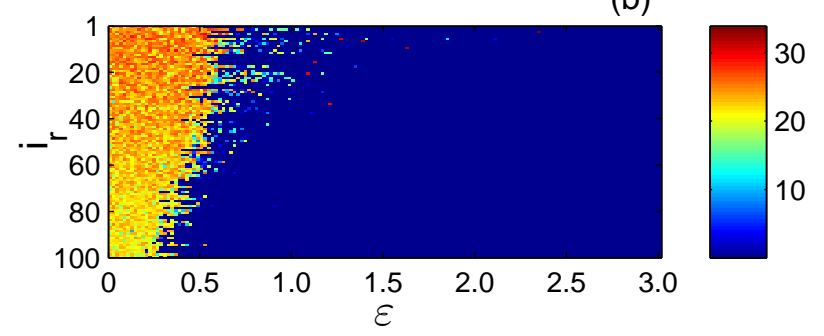

FIG. 8: (Color online) Color-maps of $d\left(\varepsilon, i_{d}\right)$ and $d\left(\varepsilon, i_{r}\right)$ in the two-dimensional parameter space $(\varepsilon, i)$ for nonidentical Lorenz oscillators on a regular network. The network has 100 nodes, and each node has 6 nearest neighboring connections.

(a)

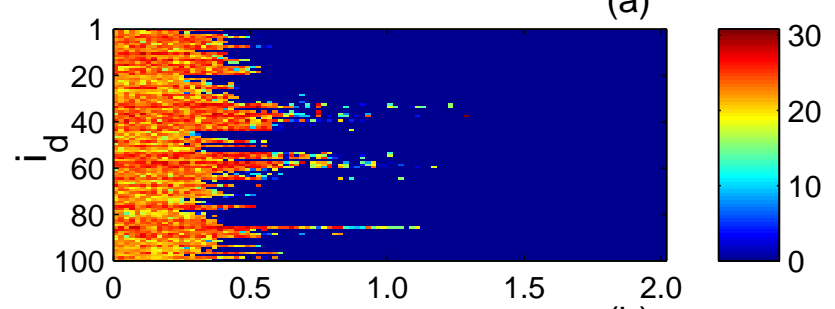

(b)

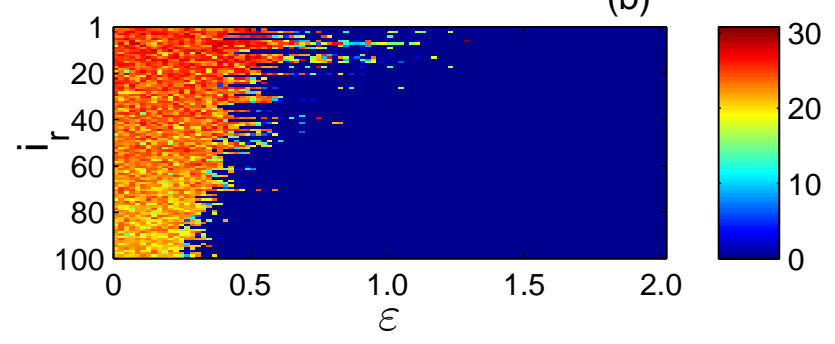

FIG. 9: (Color online) Color-maps of $d\left(\varepsilon, i_{d}\right)$ and $d\left(\varepsilon, i_{r}\right)$ in the twodimensional parameter space $(\varepsilon, i)$ for nonidentical Lorenz oscillators on a small-world network. The network is obtained by rewiring a small part of connections on a regular network with 100 nodes [9].

regular, small-world, or certain modular networks, how does the heterogeneity in the local dynamics affect GS? In the following, we study this question numerically.

We first consider a regular network of $N=100$ nodes. Each node in the network has $k_{d}=6$ connections to its nearest neighbors. As a special case, this is an exactly homogeneous network with a regular degree sequence. The local dynamics are those of Lorenz chaotic oscillators with different parameter $r_{i}$ randomly distributed in the interval $[28,38]$ for this example and for the following two examples. Figure 8 shows 


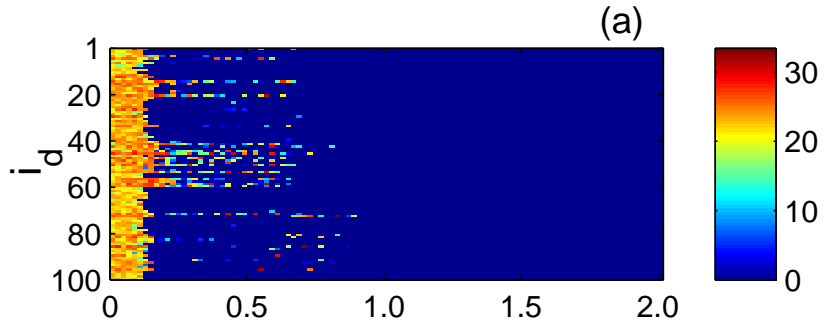

(b)

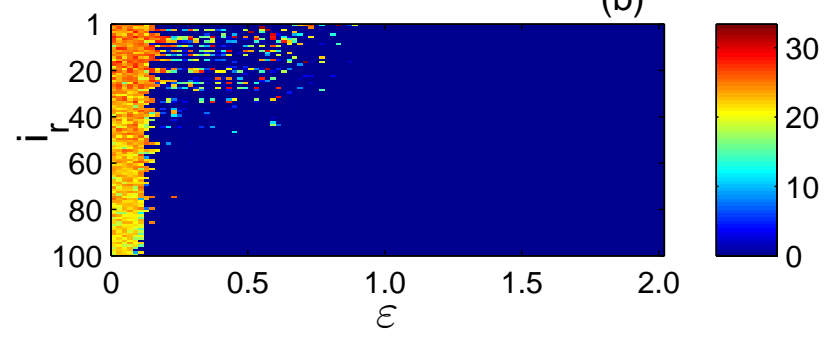

FIG. 10: (Color online) Color-maps of $d\left(\varepsilon, i_{d}\right)$ and $d\left(\varepsilon, i_{r}\right)$ in the two-dimensional parameter space $(\varepsilon, i)$ for nonidentical Lorenz oscillators on a clustered network. The network has 100 nodes which are evenly divided into 5 modules. Nodes within each module are fully connected. Any two nodes in different modules have probability $\rho=0.005$ to connect each other.

the color-maps of the local synchronization distances $d\left(\varepsilon, i_{d}\right)$ and $d\left(\varepsilon, i_{r}\right)$, where $i_{d}$ and $i_{r}$ are the node indices arranged according to the decreasing node degree and decreasing values of parameter $r_{i}$, respectively. For the Lorenz oscillator under the parameter setting in our study, we find that the larger the value of parameter $r$, the larger the largest Lyapunov exponent of the chaotic attractor. Thus, in Fig. 8, the index $i_{r}$ actually corresponds to the decreasing value of the largest Lyapunov exponent. Specifically, larger value of $i_{r}$ corresponds to a small value of $r_{i}$ so that the corresponding local dynamics is less chaotic in the sense that its largest Lyapunov exponent has a relatively smaller value. Comparing Figs. 8(a) with 8(b), we find that, when transient behaviors are disregarded, nodes whose dynamics are less chaotic require smaller value of the coupling strength to be entrained with other nodes in the network. Entrainment of more chaotic nodes requires stronger coupling. Thus the development of GS on regular network is dominated by the local dynamics.

We next consider the occurrence and development of GS on small-world networks. A representative example is shown in Fig. 9. We see from Fig. 9(a) that there is no apparent synchronization sequence of nodes according to the degree index $i_{d}$. However, as can be seen from Fig. 9(b), GS starts from nodes with smaller values of the largest Lyapunov exponent. This demonstrates that for a small-world network, heterogeneity in the local dynamics plays a dominant role in the development of GS, which is similar to the situation in regular networks.

Lastly, we investigate GS in a type of modular network. A modular network is characterized by a number of sparsely connected subnetworks, each with dense internal connections. The modular structure has been identified in biological, social, and certain technological networks [28]. For such a network, synchronization within each individual cluster can usually be achieved readily due to the dense internal connections, so the occurrence of global synchronization is particularly interesting [19]. An example of the development of GS on modular network is shown in Fig. 10. We see that global GS can be achieved despite the sparse inter-cluster connections. An interesting phenomenon is that, the development of GS does not seem to strongly depend on $i_{d}$ or $i_{r}$. Nevertheless, comparatively, it still can be found that nodes with less chaotic local dynamics are easier to be entrained with smaller coupling strength. This can be explained by noting that the degree distribution is approximately homogeneous, as a result of the sparse inter-cluster connectivity. In addition, nodes with different values of $r$ are uniformly distributed in different synchronous clusters. As a result, the effect of heterogeneity in the local dynamics is suppressed to some extent.

Based on the above studies, we can conclude that the development of GS on complex networks is the result of interaction between network topology and local dynamics when they both have heterogeneity. For heterogenous networks, such as scale-free networks, the network topology plays a leading role; while for approximately homogeneous networks, such as small-world networks and modular networks, the local dynamics is the dominant factor affecting the development and spreading of GS on networks.

\section{EFFECT OF DIFFERENT COUPLING STRATEGIES}

\section{A. The linear coupling and the normalized coupling}

We have seen that the hub nodes behave as "seeds" to develop GS in scale-free networks. The phenomenon that synchronization starts from the hub nodes in a heterogeneous network is in fact quite general, which holds for both GS and CS. Qualitatively, this can be understood by regarding the interactions among nodes as a kind of "noisy" driving within the network. In particular, since the dynamics of each individual node is chaotic, the coupling term in Eq. (1) for node $i$ is also chaotic, which can be regarded effectively as correlated noisy driving. It has been known that correlated noise can induce and/or enhance synchronization [29], PS [30], and GS as well [4]. That is, correlated noise of larger amplitude can cause oscillators under such driving to be better synchronized. For a heterogeneous network, hub nodes have significantly more links than other nodes. Since the drivings from different nodes are not entirely uncorrelated, the equivalent driving force to the hub nodes are generally stronger, facilitating their synchronization with other nodes in the network.

However, in a recent publication studying GS on networks [27], it is reported that only for a kind of special scale-free network with tree-like structure, GS is observed to develop from the hubs and then gradually spread to other nodes in the network; while for usual scale-free networks, the heterogeneity of network seems to have little effect on the development of GS in the network. In attempting to find the reason that leads to different observations in the present work and Ref. [27], we 
notice that a different coupling strategy is used in Ref. [27]. Take a coupled one-dimensional map network as an example, the coupled system in Ref. [27] is:

$$
x_{i}^{n+1}=(1-\varepsilon) f\left(x_{i}^{n}\right)+\frac{\varepsilon}{k_{i}} \sum_{j} a_{i j} f\left(x_{j}^{n}\right),
$$

where $x_{i}^{n}$ is the state variable of node $i$ at time step $n, f(x)$ is the local map, and $k_{i}$ is the degree of node $i$. This coupling scheme with each node in the network driven by the local mean field is essentially the same as the following normalized coupling strategy:

$$
\dot{\mathbf{x}}_{i}=\mathbf{F}_{i}\left(\mathbf{x}_{i}\right)-\frac{\varepsilon}{k_{i}} \sum_{j} a_{i j}\left(\mathbf{x}_{i}-\mathbf{x}_{j}\right),
$$

where notations are the same as that in Eq. (1) and Eq. (6). In this coupling scheme, the coupling strength for each node $i$ is normalized by its degree. Therefore, the effective coupling strength each node received from its network neighbors is of the same order of magnitude, regardless it is a hub or a node with very small degree. Obviously, due to the normalization, the effect of network topology on the development of GS has been almost suppressed. As a consequence, there should be no distinct difference among the coupling strength when nodes in the network achieve GS. Quantitatively, we can find that in order to obtain the same effective coupling strength, system (7) should require $\langle k\rangle$ times larger coupling strength than system (1). Here, $\langle k\rangle$ denotes the average degree of the network. Thus on an average, it can be expected that system (7) needs $\langle k\rangle$ times larger coupling strength to achieve GS in network compared with system (1).

The above heuristical considerations can be further analytically explained. By examining the coupled system (1) and its corresponding auxiliary system (2). Letting $\Delta \mathrm{x}_{i}=\mathrm{x}_{i}^{\prime}-\mathrm{x}_{i}$ and subtracting Eq. (1) from Eq. (2), we obtain

$$
\begin{aligned}
\Delta \mathbf{x}_{i} & =\mathbf{F}_{i}\left(\mathbf{x}_{i}^{\prime}\right)-\mathbf{F}_{i}\left(\mathbf{x}_{i}\right)-\varepsilon \sum_{j} a_{i j} \Delta \mathbf{x}_{i} \\
& \approx\left[\mathbf{D F}\left(\mathbf{x}_{i}\right)-\varepsilon k_{i} \mathbf{I}\right] \cdot \Delta \mathbf{x}_{i},
\end{aligned}
$$

where $\mathbf{I}$ is the $M \times M$ identity matrix with $M$ the dimension of local dynamics. We see that, for node $i$, the effective coupling strength is proportional to its degree $k_{i}$. For a fixed value of $\varepsilon$, the coupling between a hub node and its counterpart in the auxiliary system can be significantly larger than the coupling for nodes with smaller degree, leading to "earlier" synchronization between the hub nodes in the original and in the auxiliary systems. Therefore, for usual linear coupled oscillator system, the general observation is that, in a complex network with heterogeneous degree distribution, the set of hub nodes provides a skeleton around which synchronization is developed. The above analysis is also suitable for coupled system (7). In this case, there will be no factor $k_{i}$ as in Eq. (8). This implies that the effect of network topology on the development of GS in networks is eliminated.

In the following of this section, we further present two examples where the oscillators in a scale-free network are interacted with the normalized coupling strategy. The first example is coupled Lorenz oscillators described by Eq. (7), and the
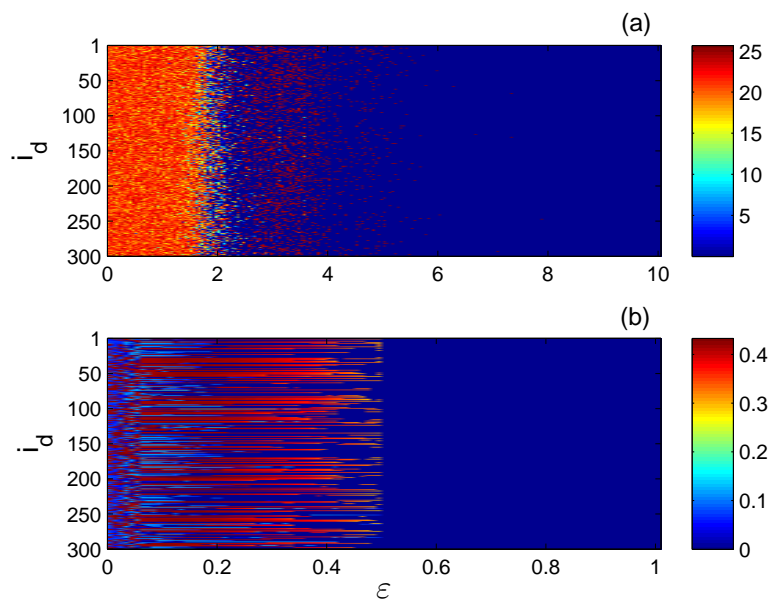

FIG. 11: (Color online) Color-maps of $d\left(\varepsilon, i_{d}\right)$ in the twodimensional parameter space $(\varepsilon, i)$, characterizing the development of GS for 300 identical Lorenz chaotic oscillators (a), and 300 identical Logistic maps (b) on a scale-free network. The network is the same as in Fig. 1. Compared with Fig. 1, the effect caused by the normalized coupling strategy is obvious.

second example is the following coupled Logistic maps:

$$
x_{i}^{n+1}=f\left(x_{i}^{n}\right)-\frac{\varepsilon}{k_{i}} \sum_{j} a_{i j}\left[f\left(x_{i}^{n}\right)-f\left(x_{j}^{n}\right)\right] .
$$

For both cases, the local dynamics are identical, i.e., $r_{i}=28$ for all Lorenz oscillators and $f(x)=4 x(1-x)$ for all Logistic maps. Figure 11 illustrates the development of GS for the above two systems on a scale-free network. The effect of different coupling strategies on the development of GS can be verified by comparing Fig. 11 with Fig. 1. For system (1), GS first occurs on the hubs and then gradually spread to other nodes; while for systems (7) and (9), GS almost simultaneously takes place on the hubs and the other nodes. Actually, there is no significant difference among all nodes on networks. By examining Fig. 1 and Fig. 11(a), it is found that the giant GS cluster forms at $\varepsilon \approx 0.5$ and $\varepsilon \approx 2$, respectively, in the two cases. In particular, the latter is approximately four times larger than the former. Note that the scale-free networks we used in Fig. 1 and Fig. 11(a) are BA model with $m_{0}=m=4$, so the average node degree $\langle k\rangle=4$. This is consistent with the theoretical analysis above.

\section{B. Coupled hybrid oscillators: competition of local dynamics}

In Sec. IV, we have studied how network topology competes with local dynamics to dominate the development of GS in complex networks when there are heterogeneity in both network topology and local dynamics. In fact, for coupled nonidentical oscillators on complex networks, competition may also occur among local dynamics if the parameter mismatches of local dynamics are large enough, or the local dynamics are physically different. By adopting the normalized coupling 


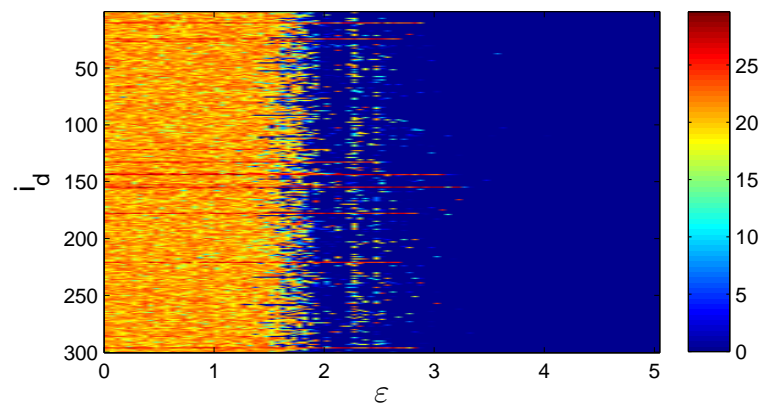

FIG. 12: (Color online) Color-map of $d\left(\varepsilon, i_{d}\right)$ in the twodimensional parameter space $(\varepsilon, i)$, characterizing 300 hybrid Lorenz chaotic oscillators on a scale-free network. The network is the BA model with $m_{0}=m=3[13]$.

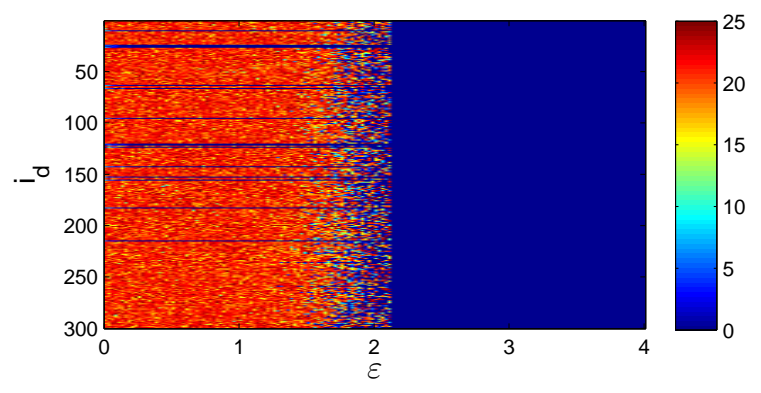

FIG. 13: (Color online) Color-map of $d\left(\varepsilon, i_{d}\right)$ in the twodimensional parameter space $(\varepsilon, i)$, characterizing 300 hybrid Lorenz and Rossler oscillators on a scale-free network. The network is the BA model with $m_{0}=m=3[13]$.

strategy, we can conveniently investigate this issue. In the following, we present two examples showing how GS is developed for coupled hybrid oscillator system in networks. By hybrid we mean that the oscillators in the network can be classified into different types which are parametrically different or physically different. In the first example, we consider a system coupled with hybrid Lorenz oscillators. To be specific, we arbitrarily select 5\% Lorenz oscillators in the network and make their parameter $r_{i}$ be randomly distributed in the interval $[30,40]$. For the rest oscillators, they are the same with $r_{i}=28$, which are significantly smaller than that of the $5 \%$ oscillators. In Fig. 12, the development of GS for such a hybrid system is illustrated on a scale-free network. It is seen that most oscillators in the network are entrained at $\varepsilon \approx 2$. However, a small number of oscillators require significantly larger coupling strength to be entrained. A careful examination of the locations of these nodes reveals that they just correspond to the 5\% oscillators with larger $r$ values. In the second example, the coupled hybrid system consists of two kinds of oscillators which have different dynamical equations. Similar to the first example, $5 \%$ nodes are randomly selected to be the identical Rossler oscillators, while the rest $95 \%$ percent nodes are the identical Lorenz oscillators with $r_{i}=28$. The dynamical equations of the Rossler oscillator read:

$$
\mathbf{F}\left(\mathbf{x}_{i}\right)=\left[-\left(y_{i}+z_{i}\right), x_{i}+0.2 y_{i}, 0.2+z_{i}\left(x_{i}-5.7\right)\right]^{T},
$$

where $\mathbf{x}_{i} \equiv\left(x_{i}, y_{i}, z_{i}\right)$ are the state variables of the Rossler oscillator. In Fig. 13, we plot the local distance of GS vs the coupling strength for all nodes in a scale-free network. It is clearly shown GS can be achieved for such a coupled hybrid system. The development of GS in network has two distinct stages: the Rossler oscillators are much easier to be entrained compared with the Lorenz oscillators. The latter needs quite larger coupling strength to achieve GS. Note that the largest Lyapunov exponent of the Lorenz oscillator is proportional to its $r$ value, and the Rossler oscillator has much smaller Lyapunov exponent than that of the Lorenz oscillator, we can conclude from the above two examples that GS usually develops from the nodes in the network where the local dynamics are less chaotic in the sense that the largest Lyapunov exponents of the local dynamics have relatively smaller values. Although our numerical simulations are carried out on a scale-free network, we believe this conclusion can still hold for other network topologies when the normalized coupling strategy is applied.

\section{SUMMARY}

In summary, we have investigated the occurrence and development of GS on various complex networks, including scale-free networks, small-world networks, random networks and modular networks. It is shown that GS generally takes place in such networks for both coupled identical oscillators and nonidentical oscillators. For coupled identical oscillators, we reveal a general bifurcation path to global CS, i.e., nonsynchronization $\rightarrow$ PGS $\rightarrow$ GS $\rightarrow$ PCS $\rightarrow$ CS. Prior to the onset of global GS (CS), there exists a coupling regime of partial GS (CS) characterized by the occurrence of a cluster of synchronous nodes. In the transient regimes, the size of the synchronous cluster usually oscillates with the increase of coupling strength, showing a dynamic scenario when the coupled system is approaching global synchronization. We find that the development of GS on complex networks depends on both network topology and local dynamics. Moreover, the specific coupling strategy also plays an important role during the evolution of synchronization. We show that under the usual linear dissipative coupling scheme, for heterogeneous networks, GS generally starts from a small number of hub nodes, and then spread to the rest nodes in the network; while for homogeneous networks, GS usually starts from the nodes whose local dynamics are less chaotic in the sense that the largest Lyapunov exponents have relatively smaller values. We further show that the effect of network topology on the development of GS can be suppressed if the coupling strengths of nodes in the network are normalized. Under such coupling scheme, the development of GS is essentially determined by the competition among different local dynamics. We demonstrate that GS can also occur in coupled systems with hybrid oscillators on complex networks, and the development of GS has distinct stages corresponding to physically different local dynamics. 
We note that, while there are extensive works on synchronization in complex networks, prior to this work the bifurcation path toward global CS via GS, the dynamical features of the development of GS, especially the interplay between the network topology and local dynamics on typical complex networks have not been investigated. Our work reveals that on complex networks, coupled oscillators may present fundamentally different synchronization regimes which deserves further study.

\section{ACKNOWLEDGMENT}

This work is supported by Temasek Laboratories at National University of Singapore.
[1] L. M. Pecora, T. L. Carroll, G. A. Johnson, D. J. Mar, and J. F. Heagy, Chaos 7, 520 (1997); A. Pikovsky, M. Rosenblum, and J. Kurths, in Synchronization: A Universal Concept in Nonlinear Science (Cambridge University Press, Cambridge, 2001); S. Boccaletti, J. Kurths, G. Osipov, D. L. Valladares, and C. S. Zhou, Phys. Rep. 366, 1 (2002).

[2] L. M. Pecora and T. L. Carroll, Phys. Rev. Lett. 64, 821 (1990); K. Pyragas, Phys. Lett. A 181, 203 (1993). L. Kocarev and U. Parlitz, Phys. Rev. Lett. 77, 2206 (1996). Y. Jiang and P. Parmananda, Phys. Rev. E 57, 4135 (1998).

[3] N. F. Rulkov, M. M. Sushchik, L. S. Tsimring, and H. D. I. Abarbane, Phys. Rev. E. 51, 980 (1995); L. Kocarev and U. Parlitz, Phys. Rev. Lett. 76, 1816 (1996); K. Pyragas, Phys. Rev. E 54, R4508 (1996); J. M. González-Miranda, ibid, 65, 047202, (2002); B. Blasius, E. Montbrió, and J. Kurths, ibid, 67, 035204(R) (2003); Z. Liu, Y.-C. Lai, and M. A. Matías, ibid, 67, 045203(R) (2003); W.-H. Kye, D.-S. Lee, S. Rim, C.-M. Kim, and Y.-J. Park, ibid, 68, 025201(R) (2003); A. Uchida, R. McAllister, R. Meucci, and R. Roy, Phys. Rev. Lett. 91, 174101 (2003); S. Guan, C.-H. Lai, and G. W. Wei, Phys. Rev. E 71, 036209 (2005).

[4] S. Guan, Y.-C. Lai, C.-H. Lai, and X. Gong, Phys. Lett. A 353, 30 (2006); S. Guan, Y.-C. Lai, and C.-H. Lai, Phys. Rev. E 73, 046210 (2006).

[5] H. D. I. Abarbanel, N. F. Rulkov, and M. M. Sushchik, Phys. Rev. E. 53, 4528 (1996).

[6] Z. Zheng, X. Wang, and M. C. Cross, Phys. Rev. E 65, 056211 (2002).

[7] S. Guan, Kun Li, and C.-H. Lai, Chaos 16, 023107 (2006).

[8] M. G. Rosenblum, A. S. Pikovsky, and J. Kurths, Phys. Rev. Lett. 76, 1804 (1996); E. Rosa, Jr., E. Ott and M. H. Hess, ibid, 80, 1642 (1998); S. Guan, C.-H. Lai, and G. W. Wei, Phys. Rev. E 72, 16205 (2005); S. Guan, X. Wang, and C.-H. Lai, Phys. Lett. A 354, 298 (2006).

[9] D. J. Watts and S. H. Strogatz, Nature 393, 440 (1998).

[10] L. F. Lago-Fernandez, R. Huerta, F. Corbacho, and J. A. Siguenza, Phys. Rev. Lett. 84, 2758 (2000); P. M. Gade and C.-K. Hu, Phys. Rev. E 62, 6409 (2000); X. F. Wang and G. Chen, Int. J. of Bifur. Chaos 12, 187 (2002); IEEE Trans. on Circ. Sys., Part I 49, 54 (2002).

[11] M. Barahona and L. M. Pecora, Phys. Rev. Lett. 89, 054101 (2002).

[12] H. Hong, M. Y. Choi, and B. J. Kim, Phys. Rev. E 65,026139 (2002).

[13] A.-L. Barabási and R. Albert, Science 286, 509 (1999).

[14] T. Nishikawa, A. E. Motter, Y.-C. Lai, and F. C. Hoppensteadt,
Phys. Rev. Lett. 91, 014101 (2003).

[15] P. N. McGraw and M. Menzinger, Phys. Rev. Lett. 72, 015101(R) (2005).

[16] E. Oh, K. Rho, H. Hong, and B. Kahng, Phys. Rev. E 72, 047101 (2005).

[17] K. Park, Y.-C. Lai, S. Gupte, and J.-W. Kim, Chaos 6, 015105 (2006); L. Huang, K. Park, Y.-C. Lai, L. Yang, and K. Yang, Phys. Rev. Lett. 97, 164101 (2006).

[18] T. Zhou, M. Zhao, G. Chen, G. Yan, and B.-H. Wang, Phys. Lett. A 368, 431 (2007).

[19] G. Guan, X. Wang, Y.-C. Lai, and C.-H. Lai, Phys. Rev. E 77, 046211 (2008).

[20] A. E. Motter, C. Zhou, and J. Kurths, Europhys. Lett. 69, 334 (2005), Phys. Rev. E 71, 016116 (2005); C. Zhou, A. E. Motter, and J. Kurths, Phys. Rev. Lett. 96, 034101 (2006); M. Chavez, D.-U. Hwang, A. Amann, H. G. E. Hentschel, and S. Boccaletti, Phys. Rev. Lett. 94, 218701 (2005).

[21] X. Wang, Y.-C. Lai, and C.-H. Lai, Phys. Rev. E 75, 056205 (2007); X. Wang, S. Guan, Y.-C. Lai, and C.-H. Lai, in process.

[22] J. G. Restrepo, E. Ott, and B. R. Hunt, Phys. Rev. E 71, 036151 (2005).

[23] J. Gómez-Gardeñes, Y. Moreno, and A. Arenas, Phys. Rev. Lett. 98, 034101 (2007).

[24] Y. Kuramoto, Chemical Oscillations, Waves and Turbulence (Springer-Verlag, Berlin,1984); S. H. Strogatz, Physica D 143, 1 (2000).

[25] L. M. Pecora and T. L. Carroll, Phys. Rev. Lett. 80, 2109 (1998).

[26] S. Guan, X. Wang, K. Li, B.-H. Wong, and C.-H. Lai, Chaos 18,013120 (2008).

[27] Y.-C. Hung, Y.-T. Huang, M.-C. Ho, and C.-K. Hu, Phys. Rev. E 77, 016202 (2008).

[28] D. J. Watts, P. S. Dodds, and M. E. J. Newman, Science 296, 1302 (2002); R. Milo, S. Shen-Orr, S. Itzkovitz, N. Kashtan, D. Chklovskii, and U. Alon, Science 298, 824 (2002); A. E. Motter, T. Nishikawa, and Y.-C. Lai, Phys. Rev. E 68, 036105 (2003).

[29] See, for example, H. Herzel and J. Freund, Phys. Rev. E 52, 3238 (1995); P. M. Gade and C. Basu, Phys. Lett. A 217, 21 (1996); E. Sanchez, M. A. Matias, and V. Perez-Munuzuri, Phys. Rev. E 56, 4068 (1997); C.-H. Lai and C. Zhou, Europhys. Lett. 43, 376 (1998); L. Kocarev and Z. Tasev, Phys. Rev. E 65, 046215 (2002).

[30] C. Zhou and J. Kurths, Phys. Rev. Lett. 88, 230602 (2002); C. Zhou, J. Kurths, I. Z. Kiss, and J. L. Hudson, Phys. Rev. Lett. 89, 014101 (2002). 